\title{
Testicular Microlithiasis: Patient Compliance in a Two- Year Follow-Up Program
}

\author{
Authors \\ Affiliations \\ M. R. Pedersen ${ }^{1}$, P. J. S. Osther ${ }^{2}$, F. B. Soerensen ${ }^{3}$, S. R. Rafaelsen \\ ${ }^{1}$ Radiology, Vejle Hospital - Part of Sygehus Lillebaelt, Vejle, Denmark \\ ${ }^{2}$ Urological Research Centre, Fredercia Hospital - Part of Sygehus Lillebaelt, Fredericia, Denmark \\ ${ }^{3}$ Clinical Pathology, Sygehus Lillebalt Vejle Sygehus, Vejle, Denmark \\ ${ }^{4}$ Department of Radiology, DCCG South Vejle Hospital, Vejle, Denmark
}

Key words

- testicular microlithiasis

testis cancer

- prevalence

follow-up

received $\quad 19.04 .2016$

accepted 21.07.2016

\section{Bibliography \\ DOI http://dx.doi.org/ \\ 10.1055/s-0042-113776 \\ Published online: \\ August 24, 2016 \\ Ultrasound International Open \\ 2016; 2: E113-E116 \\ (c) Georg Thieme Verlag KG \\ Stuttgart · New York \\ ISSN 2199-7152}

Correspondence

M. R. Pedersen, PhD Student

Radiology

Vejle Hospital - Part of Sygehus Lillebaelt

Kabbeltoft 25

7100 , Vejle

Denmark

Tel.: +45/794/06 187

malene.roland.vils.pedersen@ rsyd.dk

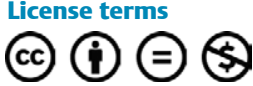

\section{Abstract}

$\nabla$

Introduction: We present a retrospective 2-year follow-up cohort of 103 men with testicular microlithiasis (TML) and discuss patient compliance and the value of surveillance.

Methods: A retrospective analysis of patients examined with scrotal ultrasonography (US) in the period from 2008 through 2010 was performed. A total of 103 men with TML were diagnosed and offered US follow-up every 6 months for 2 years. They were retrospectively analyzed regarding demographics and follow-up details, including the development of any kind of malignancy until March 2015, using the Danish Electronic Pathology Registry.

Results: The prevalence of TML was $10.3 \%$. Of the 103 men with TML, 23 (22.3\%) had TML in

\section{Introduction}

$\nabla$

Testicular microlithiasis (TML) is a relatively common finding by ultrasonography (US) of the scrotum. There is still some uncertainty whether TML should be considered an incidental benign finding or regarded as a lesion associated with testicular pre-malignancy.

A meta-analysis recently investigated the relationship between TML and testicular cancer. This study included 14 studies with more than 35000 patients and found TML significantly associated $(\mathrm{RR}=12.70, \mathrm{p}<0.001)$ with risk of developing testicular cancer [1]. The follow-up period ranged between 8.8-61.8 months, but the meta-analysis did not report the prevalence. Although some studies have found this interrelationship [2-4], other investigations have questioned the association between TML and the development of testicular malignancy $[5,6]$.

As a consequence of this controversy, the clinical management of men diagnosed with TML is car- the left testicle, 38 (36.9\%) in the right ( $\mathrm{p}=0.002$ ), and $42(40.8 \%)$ had bilateral TML. Patient compliance was low with $11.7 \%$ participating in all US follow-up examinations. 5 men presented risk factors (testicular atrophy $(\mathrm{N}=1)$ and previous testicular cancer $(\mathrm{N}=4)$ ), but no cases of testicular malignancy were found in the follow-up period.

Conclusion: The low patient compliance conflicts with the ESUR Scrotal Imaging Subcommittee guidelines that recommend scrotal US follow-up annually for TML until the age of 55 years. The fact that no cancers were found during follow-up using the pathology registry calls the value of follow-up into question.

ried out very differently from center to center. Recently, the Scrotal Imaging Subcommittee of the European Society of Urogenital Radiology (ESUR) published a consensus report on TML. ESUR presented 2 definitions of TML; 1) 5 or more TML in the whole testis, or 2) 5 or more TML per field of view. Moreover, the presence of TML in the testis, in the absence of other risk factors, is not considered an indication for annual US. The guidelines do, however, recommend US follow-up once a year until the age of 55 years in men presenting additional risk factors, such as personal/family history of testis cancer, undescended testicle, previous orchiopexy or testicular atrophy [7].

A few studies have focused on follow-up. DeCastro et al. published a 5-year follow-up study of 63 asymptomatic men with TML, one of whom (1.6\%) developed a germ cell tumor after 64 months [8]. In a recent study, Patel et al. investigated their follow-up program in a single center for a period of 14 years with 442 men diagnosed 
with TML (prevalence $4.3 \%$ ) in a population of more than 20000 men. In the follow-up period, 2 men $(0.5 \%)$ developed a testicular germ cell tumor [9]. Richenberg and Brejt performed a literature study with 2656 men referred to scrotal US, and 51 (1.9\%) were diagnosed with TML. None of these developed testicular cancer with a mean follow-up period of 33.3 months [10]. A newly published study from 2015 investigated 1249 patients with testicular cancer and found that 346 had TML. In total, 51\% (175 out of 346 ) had one or more microliths and $20 \%$ (69 out of 346) had more than 5 microliths per field of view. This investigation concluded that patients with TML may be more likely to have seminomas than embryonal components in their primary tumor [11].

The aim of this study was to investigate a 2-year follow-up program in patients with TML in order to evaluate patient compliance. The secondary aim, using the Danish Electronic Pathology Registry (i.e., Patobanken), was to investigate if any of the patients developed testicular pre-malignancy and/or manifested invasive testicular cancer.

\section{Materials and Methods \\ $\nabla$}

The study was conducted as a retrospective review of the TML follow-up program implemented at the Department of Radiology, Lillebaelt Hospital, Denmark. Men diagnosed with TML were offered scrotal US follow-up every 6 months for a period of 2 years.

TML was defined as; 1 ) classic TML with 5 or more microliths per testis or 2) limited TML with fewer than 5 microliths. All US reports were identified in the period from 2008 through 2010, using the available database (Picture Archive Communication System (PACS), Easyviz Impax Workstation, Medical Insight, Valby, Denmark).

All patients had scrotal US examination performed by experienced senior radiologists, or by experienced sonographers trained in testicular US. US was performed using one of 3 scanners: 2 kinds of 9L4 linear array transducer (Siemens, Acuson, S2000, Mountain View, CA or Siemens, Acuson, S3000, Mountain View, CA) and a 14-6 linear array transducer (Hitachi, EUB-8500, Tokyo, Japan). A series of images had been recorded in PACS at each examination as a standard procedure. All included cases were retrospectively reviewed to collect demographic data, indications for US, US results and follow-up information, including the development of testicular abnormalities. When TML was diagnosed, a follow-up scan every 6 months in a 2-year period was recommended in the US report. The US report was sent to the patient's general practitioner.

Pre-malignant and malignant testicular lesions were identified using the Danish Electronic Pathology Registry. This registry contains all histo- and cyto-pathological, SNOMED-based diagnoses of Danish citizens since the 1980s. The registry is used in the daily diagnostic routine by Danish pathologists, and it is the foundation of the Danish Cancer Registry. Moreover, the registry forms the basis for producing statistics and carrying out quality control. All men diagnosed with TML were followed until March 2015.

The study was approved by the Danish Data Protection Agency and The Regional Scientific Ethical Committees for Southern Denmark (ID\#: S-20120144).

\section{Results}

$\nabla$

A total of 1005 patients with scrotal US examinations were retrieved from PACS and 103 adult men and 2 boys were diagnosed with TML. The 2 boys were excluded from the study. The clinical indications for the baseline US are summarized in $\bullet$ Fig. $\mathbf{1}$, testicular pain being the most frequent cause of referral to scrotal US.

The prevalence of TML was $10.3 \%$, with a mean age of 42 (range 19-80) years. In addition to TML, US diagnoses are specified in 0 Table 1.

A total of 23 men (22.3\%) had TML in the left testicle, 38 (36.9\%) in the right $(\mathrm{p}=0.02)$, and $42(40.8 \%)$ had bilateral TML. $\odot$ Table 2 shows the distribution of TML at baseline US.

All 103 men were offered entry into the US follow-up program by the radiologist at the baseline US scan. At the subsequent US scan, the radiologist offered a new US scan after a time period of 6 months. However, only 12 men (11.7\%) participated in the full 2-year follow-up program ( $\bullet$ Fig. 2 ). 6 of these 12 men (50\%) had 1-4 TML, 3 (25\%) had 5-10 TML, 2 (16.7\%) had multiple TML, and 1 man (8.3\%) diagnosed with 1-4 TML at baseline US experienced disappearance of the TML at the final US follow-up.

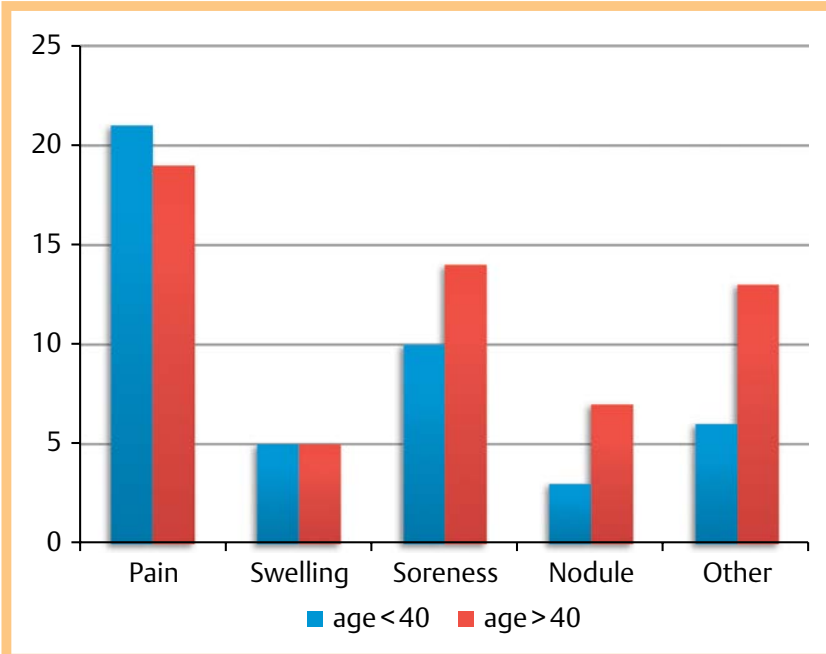

Fig. 1 Clinical indications in 103 men with testicular microlithiasis for referral to scrotal ultrasonography.

Table 1 Overview of additional diagnoses from the scrotal ultrasonography (US) diagnoses at the baseline scans in the 103 men with testicular microlithiasis (TML).

\begin{tabular}{|lccc|}
\hline Diagnosis & $\mathbf{4 0}$ years & $\mathbf{4 0}$ years & Total \\
& $\mathbf{4 6}$ men & $\mathbf{5 7}$ men & $\mathbf{1 0 3} \mathbf{~ m e n}$ \\
Spermatocele & 19 & 22 & 41 \\
\hline Hydrocele & 4 & 12 & 16 \\
\hline Varicocele & 5 & 14 & 19 \\
\hline Epididymitis & 3 & 2 & 5 \\
\hline Testicular atrophy & 1 & 3 & 4 \\
\hline Inguinal hernia & - & 5 & 5 \\
\hline Previously orchiectomy & 1 & 1 & 2 \\
\hline Granuloma & - & 2 & 2 \\
\hline Suspicion of testicular tumor or & 5 & 1 & 6 \\
carcinoma in situ & & & 28 \\
\hline No additional US diagnosis & 14 & 14 & \\
\hline Some men had more than one additional diagnosis & & \\
\hline
\end{tabular}

Some men had more than one additional diagnosis 
Table 2 Grading of testicular microlithiasis (TML) at the baseline scrotal ultrasonography (US) in men with bilateral TML.

\begin{tabular}{|lc} 
Grading of TML at baseline ultrasonography & Number of men (\%) \\
Some $(1-4)$ & $60(58.3 \%)$ \\
\hline Several $(5-10)$ & $25(24.3 \%)$ \\
\hline Multiple $(11+)$ & $15(14.5 \%)$ \\
\hline Number not reported & $3(2.9 \%)$ \\
\hline Total & $103(100 \%)$
\end{tabular}

The numbers refer to the testicle with the highest amount of TML

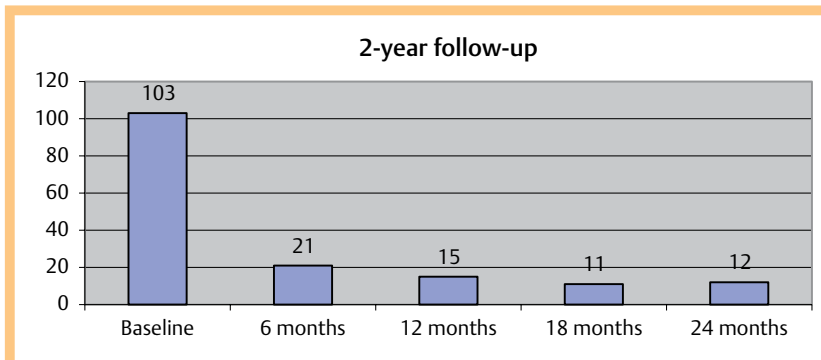

Fig. 2 Number of men participating in the follow-up program.

Table 3 Risk factors encountered among 103 men diagnosed with testicular microlithiasis (TML).

\begin{tabular}{|lcc}
\hline Risk factors & $\begin{array}{l}\text { Number } \\
\text { of patients } \\
\text { with TML }\end{array}$ & $\begin{array}{l}\text { Number of patients } \\
\text { developing testicular can- } \\
\text { cer during US follow-up }\end{array}$ \\
\hline Testicular atrophy & 1 & 0 \\
\hline Previous testicular cancer & 4 & $0^{*}$ \\
\hline * One man with previously diagnosed, right-sided testicular cancer in 2006 was diag- \\
nosed with cancer in the remaining left testicle at the baseline ultrasonography (US)
\end{tabular}

A total of 13 of the 103 men were referred to further investigation at the Department of Urology, Lillebaelt Hospital, Denmark, 10 at the baseline US and 3 men later in the follow-up US program. These men were referred either because of multiple TML $(\mathrm{N}=5)$, the clinical suspicion of carcinoma in situ $(\mathrm{N}=3)$, testicular cancer $(\mathrm{N}=3)$, atrophy $(\mathrm{N}=1)$ or varicocele $(\mathrm{N}=1) .2$ of the 13 men, referred at baseline US, had a final histopathological diagnosis of testicular cancer. According to the clinical records, one of these patients had previously been diagnosed with testicular cancer in the right testicle in 2006, and was now diagnosed with cancer in the left testicle, in which baseline US had shown several TML. The other patient, diagnosed with cancer in the right testicle at baseline US, had between 5-10 bilateral TML. After the right-sided orchiectomy, the patient never returned to the US follow-up program. 3 other men from the referral group of 13 men underwent testicular biopsy on the clinical suspicion of pre-malignant or malignant testicular lesion. The histopathological examination of their biopsies showed, however, no signs of malignancy.

Searching the Danish Electronic Pathology Registry revealed that none of the remaining 101 men with TML were diagnosed with testicular cancer in the follow-up period until March 2015. 3 men with a mean age of 62.2 (range 50-70) years were diagnosed with prostate cancer in 2009, 2010 and 2011, respectively. Moreover, one man was diagnosed with pancreatic cancer in 2012. 8 patients had undergone sterilization before 2008 , i.e., before inclusion in the study at baseline US. None of these revealed any pathology in the follow-up period.

5 men with recognized risk factors of developing testicular cancer were detected in the study ( $\bullet$ Table 3). Apart from the 2 men diagnosed with testicular cancer at baseline US, 2 other men had previously, before inclusion in the present study, been diagnosed with testicular cancer, and none developed testicular cancer in the follow-up period. All 103 patients could be identified in the Danish Electronic Pathology Registry.

\section{Discussion}

The TML prevalence of $10.3 \%$ in the present study population of 1005 patients ranges within the upper part of previously reported TML prevalence. A literature survey documents an estimated prevalence between 0.6-9.0\% [12-15]. Differences in study population, e.g., size and age distribution, and research focus may offer explanations regarding the variations in TML prevalence in published studies.

The priority given by the 103 men with TML to participate in the offered US follow-up schedule seems rather low. It was the radiologist's responsibility to offer US follow-up to the patients. If this recommendation was only offered orally, this may cause a lower participation rate in the follow-up program. Moreover, if symptoms were absent after 6 months, there is a potential risk that patients tend to skip continuation of the follow-up. US examinations in Denmark are free of payment, and in our Department of Radiology the patients are offered direct access to US on a day-to-day basis. Thus, no financial burden hinders the men from participating.

It will most likely be a clinical challenge to increase compliance in this group of men regarding their participation in annual US. Moreover, the follow-up participation rate will probably be very low, if the annual scrotal US continues over an extended time period until the age of 55 years. This prolonged "screening program" will also produce unnecessary patient distress and anxiety, which may not correspond with any real clinical benefit for the patient. Consequently, one may question the clinical relevance, patient compliance and cost-benefit of the US follow-up program suggested by the ESUR Scrotal Imaging Subcommittee. Some studies have reported that bilateral TML increases the risk of developing testicular cancer $[16,17]$, but so far no clear association has been proved. We found $42(40.8 \%)$ patients with bilateral TML, and one of those was diagnosed with testicular cancer at the baseline US.

13 men were referred to further investigation at the Department of Urology, and 2 were diagnosed with testicular cancer at their baseline US, whereas we did not detect any men in the study group who developed testicular neoplasia within the US followup period. All ultrasound reports of the 103 patients were subsequently reviewed. Besides the 13 men referred to further investigation at the Department of Urology, none of the remaining men in the study had a diagnosis of testicular neoplasia according to the Danish Electronic Pathology Registry. The coverage and precision of classification in this national registry are extremely high, and it can be concluded that none of the investigated men developed testicular germ cell neoplasia within a minimal time frame of 50 months (range: 50-86 months) after conclusion of the US follow-up program. Thus, we detected one man with atrophic testis and 4 men with testicular cancer diag- 
nosed previously or at baseline US. According to the Danish Electronic Pathology Registry, none of these men developed testicular germ cell neoplasia in the follow-up until March 2015. The incidence of testicular cancer is more than 3 times as high in men aged 15-35 years than among men above the age of 50 years. The age distribution of our study population has a mean of 42 years. Accordingly, our study population is representative and includes the high-risk group. Moreover, we examined the diagnostic differences between men in 2 age groups. In the group of men $\leq 40$ years, the diagnostic suspicion of malignant testicular tumor/carcinoma in situ was detected 5 times, as compared to only one time in the age group $>40$ years. Because our study population only consists of 103 men with TML, we did not expect any of them to develop testicular cancer.

A limitation of the present study is the lack of a clear definition of TML. The ESUR guidelines state 2 definitions - 5 TML or more in the testis or per field of view. The cut-off point of 5 TML has been broadly accepted in the literature, but on the other hand many studies make use of the definition classic or limited TML [18-21]. One could argue that the presence of a risk factor, and not the number of TML, should determine if the follow-up program should be offered. Therefore, we have chosen to implement both the limited and the classic definition of TML. A recent study investigated 346 testicular tumors and found that $51 \%$ had one or more microliths per image [11]. This finding may offer an argument for not disregarding men with limited TML. Another limitation of our retrospective study is that we did not discriminate between age groups, and high- vs. low-risk patients. The reason for this is that the study was initiated prior to the ESUR guidelines [7], which explains the inclusion of some men older than the recommended limit of 55 years.

Our main focus was to study patient compliance, and the secondary aim was to disclose any development of testicular malignancy in a 2-year follow-up program. The relatively short observation period for developing testicular malignancy (50-86 months) after baseline US represents a limitation of our study. On the other hand, a recent meta-analysis had an even shorter follow-up period ranging between 8.8 and 61.8 months [1]. The strength of our study was the opportunity to follow all men diagnosed with TML within the framework of the Danish Electronic Pathology Registry simply by using the unique Central Personal Registration Number, which is provided to every citizen in Denmark. This registration number is linked to all medical records and official registries and can thus be used e.g., to find information on any diagnosed cancer.

In conclusion, our findings suggest low patient compliance for participating in a scrotal US follow-up program in the event of diagnosed TML. Accordingly, annual follow-up US may be difficult to implement in clinical practice. Furthermore, this study cannot discern any correlation between TML and later occurrence of testicular germ cell neoplasia.

\section{References}

1 Wang T, Liu L, Luo J et al. A meta-analysis of the relationship between testicular microlithiasis and incidence of testicular cancer. Urol J 2015; 12: 2057-2064

2 Derogee M, Bevers RFM, Prins HJ et al. Testicular microlithiasis, a premalignant condition: prevalence, histopathologic findings, and relation to testicular tumor. Urology 2001; 57: 1133-1137

3 Sakamoto H, Shichizyou T, Saito K et al. Testicular microlithiasis identified ultrasonographically in Japanese adult patients: prevalence and associated conditions. Urology 2006; 68: 636-641

4 Thomas D, Vlachopapadopoulou E, Papadakis $V$ et al. Testicular microlithiasis in siblings: clinical implications. Pediatr Radiol 2008; 38: 688-690

5 Costabile RA. How worrisome is testicular microlithiasis? Curr Opin Urol 2007; 17: 419-423

6 Goede J, Hack WWM, van der Voort-Doedens LM et al. Prevalence of testicular microlithiasis in asymptomatic males 0 to 19 years old. J Urol 2009; 182: 1516-1520

7 Richenberg J, Belfield J, Ramchandani P et al. Testicular microlithiasis imaging and follow-up: guidelines of the ESUR scrotal imaging subcommittee. Eur Radiol 2015; 25: 323-330

8 DeCastro BJ, Peterson AC, Costabile RA. A 5-year followup study of asymptomatic men with testicular microlithiasis. J Urol 2008; 179: $1420-1423$

9 Patel KV, Navaratne S, Bartlett E et al. Testicular Microlithiasis : Is sonographic surveillance necessary? Single centre 14 year experience in 442 patients with testicular microlithiasis. Ultraschall Med 2016; 37: $68-73$

10 Richenberg J, Brejt $N$. Testicular microlithiasis: Is there a need for surveillance in the absence of other risk factors? Eur Radiol 2012; 22: 2540-2546

11 Sharmeen F, Rosenthal MH, Wood MJ et al. Relationship between the pathologic subtype/initial stage and microliths in testicular germ cell tumors. J Ultrasound Med 2015; 34: 1977-1982

12 Cast JE, Nelson WM, Early AS et al. Testicular microlithiasis: prevalence and tumor risk in a population referred for scrotal sonography. AJR 2000; 175: 1703-1706

13 Peterson AC, Bauman JM, Light DE et al. The prevalence of testicular microlithiasis in an asymptomatic population of men 18 to 35 years old. J Urol 2001; 166: 2061-2064

14 Bach AM, Hann LE, Hadar $O$ et al. Testicular microlithiasis: What is its association with testicular cancer? Radiology 2001; 220: 70-75

15 Skyrme RJ, Fenn NJ, Jones AR et al. Testicular microlithiasis in a UK population: its incidence, associations and follow-up. BJU Int 2000; 86: 482-485

16 van Casteren NJ, Looijenga LH, Dohle GR. Testicular microlithiasis and carcinoma in situ overview and proposed clinical guideline. Int J Androl 2009; 32: 279-287

17 Sanli 0 , Kadioglu A, Atar $M$ et al. Grading of classical testicular microlithiasis has no effect on the prevalence of associated testicular tumors. Urol Int 2008; 80: 310-316

18 Middleton WD, Teefey SA, Santillan CS. Testicular microlithiasis: prospective analysis of prevalence and associated tumor. Radiology 2002; 224: 425-428

19 Korde LA, Premkumar A, Mueller C et al. Increased prevalence of testicular microlithiasis in men with familial testicular cancer and their relatives. Br J Cancer 2008; 99: 1748-1753

20 Vachon L, Fareau GE, Wilson MG et al. Testicular microlithiasis in patients with down syndrome. J Pediatr 2006; 149: 233-236

21 Lam DL, Gerscovich EO, Kuo MC et al. Testicular Microlithiasis. J Ultrasound Med 2007; 26: 867-873 